\title{
Outcome and predictors for successful resuscitation in the emergency room of adult patients in traumatic cardiorespiratory arrest
}

\author{
J. Zwingmann ${ }^{1 *}$, R. Lefering ${ }^{2}$, M. Feucht ${ }^{1}$, N. P. Südkamp ${ }^{1}$, P. C. Strohm ${ }^{3}$ and T. Hammer ${ }^{1}$
}

\begin{abstract}
Background: Data of the TraumaRegister DGU ${ }^{\circledR}$ were analyzed to derive survival rates, neurological outcome and prognostic factors of patients who had suffered traumatic cardiac arrest in the early treatment phase.

Methods: The database of the TraumaRegister DGU ${ }^{\circledR}$ from 2002 to 2013 was analyzed. The main focus of this survey was on different time points of performed resuscitation.

Descriptive and multivariate analyses (logistic regression) were performed with the neurological outcome (Glasgow Outcome Scale) and survival rate as the target variable. Patients were classified according to CPR in the prehospital phase and/or in the emergency room (ER). Patients without CA served as a control group. The database does not include patients who required prehospital CPR but did not achieve ROSC.

Results: A total of 3052 patients from a total of 38,499 cases had cardiac arrest during the early post-trauma phase and required CPR in the prehospital phase and/or in the ER. After only prehospital resuscitation $(n=944)$ survival rate was $31.7 \%$, and $14.7 \%$ had a good/moderate outcome. If CPR was required in the ER only $(n=1197)$, survival rate was $25.6 \%$, with a good/moderate outcome in $19.2 \%$ of cases. A total of $4.8 \%$ in the group with preclinical and ER resuscitation survived, and just $2.7 \%$ had a good or moderate outcome. Multivariate logistic regression analysis revealed the following prognostic factors for survival after traumatic cardiac arrest: prehospital CPR, shock, coagulopathy, thorax drainage, preclinical catecholamines, unconsciousness, and injury severity (Injury Severity Score).

Conclusions: With the knowledge that prehospital resuscitated patients who not reached the hospital could not be included, CPR after severe trauma seems to yield a better outcome than most studies have reported, and appears to be more justified than the current guidelines would imply. Preclinical resuscitation is associated with a higher survival rate and better neurological outcome compared with resuscitation in the ER. If resuscitation in the ER is necessary after a preclinical performed resuscitation the survival rate is marginal, even though $56 \%$ of these patients had a good and moderate outcome. The data we present may support algorithms for resuscitation in the future.
\end{abstract}

Abbreviations: AIS, Abbreviated Injury Severity Score; AUC, AUC - Academy for Trauma Surgery (AUC - Akademie der Unfallchirurgie GmbH); Cl, Confidence interval; CPR, Cardiopulmonary resuscitation; DGU, Deutsche Gesellschaft für Unfallchirurgie; ECC, Emergency cardiac care; EMS, Emergency medical services; ER, Emergency room; GCS, Glasgow Coma Scale; GOS, Glasgow Outcome Scale; ICU, Intensive care unit; ISS, Injury Severity Score; NISS, New Injury Severity Score; OHCA, Out-of-hospital cardiac arrest; ROSC, Return of spontaneous circulation; RR, Riva Rocchi; Sektion NIS, Notfall-, Intensivmedizin und Schwerverletztenversorgung; TCPA, Traumatic cardiopulmonary arrest; TCRA, Traumatic cardiorespiratory arrest; TR-DGU, TraumaRegister DGU ${ }^{\oplus}$

\footnotetext{
*Correspondence: joern.zwingmann@uniklinik-freiburg.de

'Department of Orthopedics and Trauma Surgery, Freiburg University

Hospital, Hugstetter Str. 55, 79098 Freiburg, Germany

Full list of author information is available at the end of the article
} 


\section{Background}

Patients suffering traumatic cardiorespiratory arrest (TCRA) are generally reported to have a poor outcome [1-12]. A recent systematic review revealed that children appear to have a better chance of survival after resuscitation than adults after suffering out-of-hospital traumatic cardiac arrest, but also that they tend to have a poorer neurological outcome at discharge [13]. Cardiopulmonary resuscitation (CPR) in children after severe trauma seems to yield a better outcome than in adults, and according to a recent study, appears to be more justified than the current guidelines would imply. Resuscitation in the emergency room (ER) was in that study associated with better neurological outcomes compared with resuscitation in a preclinical context or in both the preclinical phase and the ER [14].

The epidemiology of mortality following a polytrauma suggests that as many as $34 \%$ of traumatic deaths occur before hospital arrival [15]. In a German study even 58.7 $\%$ of trauma victims died before reaching a hospital [16]. The same authors investigated in another study that 15.2 $\%(n=40)$ of traumatic deaths were classified as preventable [17].

Despite advances in medical treatment and algorithms, only marginal survival rates ( 0 to $2 \%$ ) have been reported for blunt trauma patients who arrive at a trauma centre with no signs of life $[4,8,12,18,19]$. Unfortunately, many of these survivors suffer from severe permanent neurological disability $[6,12]$.

The National Association of Emergency Medical Service (EMS) Physicians/American College of Surgery Committees on Trauma produced guidelines in 2003 on the withholding or termination of resuscitation efforts in out-of-hospital cardiac arrest (OHCA) [19]. Since those guidelines were published, at least two articles have described higher survival rates $[1,20]$ and one reports a possible deviation from them [20].

In a German study, a tCPR algorithm was introduced, including chest/pericardial decompression, external pelvic stabilization and external bleeding control. The authors showed in their study that prehospital trauma management has the highest potential to improve tCPR and survival [21].

On the other hand, another recent article supported the current guidelines on the withholding or termination of resuscitation of patients in prehospital traumatic cardiopulmonary arrest TCPA [22].

The risk factors associated with different time points of resuscitation (preclinical, ER, or at both time points) were recently published in a huge pediatric population. Here, the population that was resuscitated in the ER revealed the lowest mortality and best outcomes [14]. Not much is known about the prognostic factors for successful resuscitation in the ER of adults suffering TCPA.
The aim of this study was to identify the risk factors and analyze the outcomes of an adult population in the TraumaRegister DGU ${ }^{\bullet}$ (TR-DGU) who were resuscitated in the early post-traumatic phase (prehospital and/or ER).

The TR-DGU collects data and provides a sound basis for even rare events in severely injured trauma patients. The purpose of this study was to analyze the subgroup of patients who required cardiopulmonary resuscitation in the early phase after trauma but were admitted to hospital. Specifically, survival rates and prognostic factors were investigated in this patient group. Beyond survival, the neurological outcome assessed by the Glasgow Outcome Scale (GOS) was considered as well.

\section{Methods}

\section{TraumaRegister DGU ${ }^{\circledR}$}

The TraumaRegister DGU ${ }^{\bullet}$ (TR-DGU) of the German Trauma Society (Deutsche Gesellschaft für Unfallchirurgie, DGU) was founded in 1993 [23]. The aim of this multi-centre database is an anonymous and standardised documentation of severely injured patients for the purpose of quality control. Data are collected prospectively at four consecutive time periods from the site of the accident until discharge from hospital: (A) prehospital phase, (B) emergency room and initial surgery, (C) intensive care unit and (D) at discharge. This documentation records detailed information on demographics, injury pattern, comorbidities, pre- and in-hospital management, course on the intensive care unit, relevant laboratory findings including data on transfusions, and each individual's outcome. The inclusion criterion is admission to hospital via the emergency room with subsequent intensive care unit (ICU) care. Patients who reach the hospital with vital signs and die before admission to the ICU are included as well.

The infrastructure for documentation, data management, and data analysis is provided by the Academy for Trauma Surgery (AUC - Akademie der Unfallchirurgie $\mathrm{GmbH})$, a company affiliated with the German Trauma Society. The scientific leadership is provided by the Committee on Emergency Medicine, Intensive Care and Trauma Management (Sektion NIS) of the German Trauma Society. The participating hospitals submit their data to a central database via a web-based application. Scientific data analysis is approved according to a peerreview procedure established by the Sektion NIS.

Most of the participating hospitals are located in Germany (90\%), but a rising number of hospitals in other countries have been contributing data as well (these include currently Austria, Belgium, China, Finland, Luxemburg, Slovenia, Switzerland, the Netherlands, and United Arab Emirates). About 35,000 cases from over 600 hospitals are currently being entered into the database per year. 
Participation in the TR-DGU is voluntary. For certified hospitals associated with the TraumaNetzwerk DGU ${ }^{\circ}$, however, participation is obligatory for reasons of quality assurance.

Data anonymity for scientific analyses is guaranteed for both the individual patient and participating hospital. [23-25].

The present study is in line with the publication guidelines of the TraumaRegister $\mathrm{DGU}^{\circ}$ and registered as a TR-DGU project ID 2014-023.

\section{Patients}

We analyzed the 2013 database from 2002 to 2013. Primary admitted adult patients (age $\geq 16$ ) from Europe with Injury Severity Score (ISS) $\geq 16$ points and available information on CPR (done, or not) both in the prehospital phase and during emergency room (ER) treatment qualified for analysis. Patients transferred in from other hospitals were excluded since prehospital information was missing. Patients declared dead at the accident scene and not transported to a hospital were not recorded in the TR-DGU. The main focus of this survey was the group of patients in cardiac arrest resuscitated in the ER and in the prehospital phase.

A patient's death can be pronounced outside a hospital and/or clinic setting in Germany, thus the patient is not transported to a medical setting to be declared dead, as happens in some other countries. This requires the diagnosis of at least one definitive sign of death. Patients without return of spontaneous circulation (ROSC) after cardiac arrest were not included in the TR-DGU, according to our inclusion criteria for the registry. This holds true also for patients admitted to the hospital with ongoing CPR but without subsequent ROSC. The registry was established for quality assessment in the acute care hospital and thus did and does not document prehospital deaths.

\section{Statistical analysis}

We performed a descriptive analysis of patients with and without resuscitation in the ER. Then a multivariate logistic regression analysis with resuscitation in the ER as a dependent variable was done to identify independent predictors for survival by calculating odds ratios for each factor. Odds ratios are presented with $95 \%$ confidence intervals $(95 \% \mathrm{CI})$. Nagelkerke's $\mathrm{R}^{2}$ was used as an overall goodness-of-fit measure for the model.

The Glasgow Outcome Scale (GOS) was used to classify patients with good or moderate (GOS 4-5) and dead/bad outcome (GOS 1-3) [26]. Furthermore, we carried out a multivariate logistic regression analysis with bad outcome as a dependent variable. Moreover all parameters for the Trauma-Associated Severe Haemorrhage (TASH) score (a score that reliably predicts the probability for mass transfusion after multiple trauma) [27] and coagulopathy (presence of abnormal coagulation parameters upon the patient's arrival); i.e. prothrombin time test, Quick's value $<70 \%$ and/or platelets $<100,000 / \mathrm{ml}$ ) [28] and acidosis (base excess $\leq-6$ ) were analysed.

Differences between the groups were evaluated by applying chi-squared test for categorical variables and Mann-Whitney $U$ test for quantitative and ordinal measures.

Statistical significance was defined as $p<0.05$. Statistical analysis was performed using SPSS Version 20.0 (IBM Corp., Armonk, NY, USA).

\section{Results}

In total 38,499 patients met the inclusion criteria. A prehospital cardiac arrest with subsequent CPR was observed in 1855 patients. These patients had return of spontaneous circulation (ROSC) and were transported to a hospital; the number of cases with attempted but unsuccessful CPR is not documented in the TR-DGU.

The TR-DGU only includes patients who arrived at the hospital. Patients who were dead/who died in the field or patients or without ROSC after cardiac arrest were not included in the TR-DGU, according to our inclusion criteria for the registry.

Among these cases, 944 patients did not require further cardiac massage in the ER while 911 cases again received CPR in the ER. The total number of patients who required CPR in the ER was 2108 (5.5\% of all admitted patients). Among them were 1197 patients (3.1\%) who were only resuscitated in the ER. The patient subgroups are illustrated in Fig. 1.

Epidemiological data are presented in Table 1 covering 2108 patients, who were treated in the ER after only CPR in the ER ( $\mathrm{n}=1197)$ and after prehospital and additional CPR in the ER ( $\mathrm{n}=911)$. The "Control group" comprises 36,391 patients who were treated in the ER (including 35,477 patients without any CPR and 944 patients after prehospital CPR).

\section{Mortality}

The hospital mortality rate of all resuscitated patient groups was a total of $83.4 \%(n=2545)$ with patients after preclinical resuscitation alone only amounted to $68.3 \%(\mathrm{n}=645)$, after ER resuscitation only amounted to $74.4 \%(\mathrm{n}=891)$ and patients who required preclinical and $\mathrm{ER}$ resuscitation had the highest mortality rate with $95.2 \%(\mathrm{n}=867)$.

A total of 1755 patients died after CPR in the ER; there was a mean time to death of 2.3 days (SD 5.8; median 1). Of these 1558 (73.9\% of all, $88.6 \%$ of those who died) died within the first 24 hours after admission. The 350 surviving patients stayed on the ICU for 20.8 


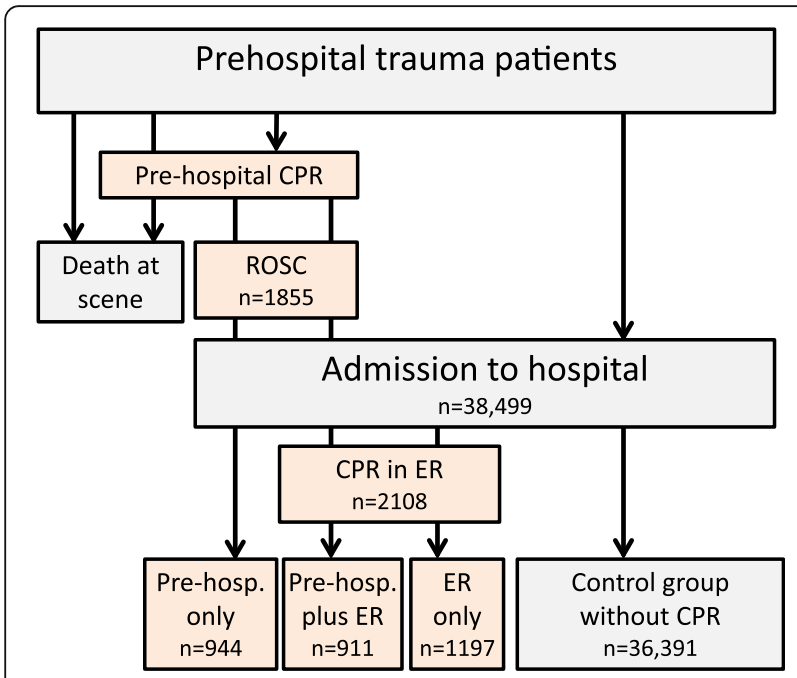

Fig. 1 In total 38,499 patients met the inclusion criteria. A prehospital cardiac arrest with subsequent CPR was observed in 1855 patients. These patients had return of spontaneous circulation (ROSC) and were transported to a hospital; the number of cases with attempted but unsuccessful CPR is not documented in the TR$\mathrm{DGU}^{\oplus}$. Among these cases, 944 patients did not require further cardiac massage in the ER while 911 cases again received CPR in the ER. The total number of patients who required CPR in the ER was 2108 (5.5\% of all admitted patients). Among them were 1197 patients (3.1\%) who were only resuscitated in the ER. The patient subgroups are illustrated in Fig. 1. CPR cardiopulmonary resuscitation, ER emergency room, TR-DGU TraumaRegister DGU ${ }^{\oplus}$

days (SD 10.0, median 16.5); their hospital stay was 42.0 days (SD 34.2; median 33.5).

Results of multivariate logistic regression analysis with resuscitation in the ER as a dependent variable (Nagelkerke's $\left.R^{2}=0.30\right)$ are shown in Table 2 .

The highest risk for mortality in this model was associated with preclinical resuscitation (OR 5.74), followed by shock on admission (OR 3.49), coagulopathy (OR 2.18), preclinical thorax drainage (OR 1.54), preclinical catecholamines (OR 1.52), mild hypotension with preclinical blood pressure $\leq 90 \mathrm{mmHG}$ (OR 1.35), preclinical GCS $\leq 8$ (OR 1.28), relevant injuries of the extremities (OR 1.27), the thorax (OR 1.26), and the abdomen (OR 1.23). The overall injury severity is also associated with in-hospital CPR.

\section{Neurological outcome}

Results of the Glasgow Outcome Scale (GOS) [26] after resuscitation in the preclinical phase and/or in the ER are based on data from 3052 patients. Table 3 displays the Glasgow Outcome Scale (GOS) groups of resuscitated patients.

We defined a good or moderate outcome as GOS 5 and 4 and this was analysed in patients without any resuscitation in 68.5. After only preclinical resuscitation the survival rate was with $31.7 \%$ the highest and $14.7 \%$ had a good or
Table 1 Epidemiological data are presented covering 2108 patients, who were treated in the ER after only CPR in the ER ( $n=$ $1197)$ and after prehospital and additional CPR in the ER $(n=911)$

\begin{tabular}{lll}
\hline & $\begin{array}{l}\text { Control } \\
\text { group } \\
\mathrm{n}=36391\end{array}$ & $\begin{array}{l}\text { CPR in } \\
\text { the ER } \\
\mathrm{n}=2108\end{array}$ \\
\hline Age (years) & $48.1 \pm 20.1$ & $49.0 \pm 21.0$ \\
ISS & $27.9 \pm 11.0$ & $42.0 \pm 18.1$ \\
Male/female & $27.1 \% / 72.9$ & $29.9 \% / 70.1$ \\
& $\%$ & $\%$
\end{tabular}

Trauma cause

Car

Motorcycle

$27.4 \%$

$29.2 \%$

Bicycle

$14,0 \% \quad 13.8 \%$

Pedestrian

$8.4 \%$

$7.1 \%$

Pedestrian

$7.8 \%$

$13,0 \%$

High fall ( $\geq 3 \mathrm{~m}$ )

$19.1 \%$

$19.7 \%$

Low fall

$14.5 \% \quad 4.7 \%$

Others

$8.9 \%$

$12.6 \%$

Traffic accident

$41.5 \%$

$35.2 \%$

Blunt trauma

$95.7 \%$

$92.1 \%$

Penetrating trauma

$4.3 \%$

$7.9 \%$

AIS head $\geq 3$

$56.8 \%$

$58.6 \%$

AIS thorax $\geq 3$

$56.2 \%$

$73.6 \%$

AlS abdomen $\geq 3$

$18.4 \%$

$31.8 \%$

AlS extremities $\geq 3$

$33.9 \%$

$46.5 \%$

Preclinical volume $(\mathrm{ml})$

$1107 \pm 864$

$1601 \pm 1155$

Time from accident to hospital admission $68.4 \pm 29.3$

$64.0 \pm 27.2$ (min)

\begin{tabular}{|c|c|c|}
\hline Preclinical resuscitation & $2.6 \%$ & $56.8 \%$ \\
\hline Preclinical GCS $\leq 8$ & $31.9 \%$ & $70.6 \%$ \\
\hline Preclinical shock (BP $\leq 90 \mathrm{mmHG})$ & $17.0 \%$ & $59.9 \%$ \\
\hline Preclinical intubation & $49.6 \%$ & $84.8 \%$ \\
\hline Preclinical transfusion of catecholamines & $9.1 \%$ & $53.3 \%$ \\
\hline Preclinical thoracic drainage & $5.4 \%$ & $18.0 \%$ \\
\hline $\begin{array}{l}\text { Mild hypotension on admission } \\
\text { (BP } \leq 90 \mathrm{mmHG} \text { ) }\end{array}$ & $13.4 \%$ & $63.6 \%$ \\
\hline $\begin{array}{l}\text { Blood transfusion: number of } \\
\text { packed red blood cell units }\end{array}$ & $21.9 \%$ & $63.6 \%$ \\
\hline TASH score & $6.2 \pm 5.0$ & $13.8 \pm 6.0$ \\
\hline Hemoglobin ER & $12.1 \pm 2.6$ & $9.1 \pm 3.6$ \\
\hline Quick's value ER & $79.1 \pm 22.9$ & $50.7 \pm 28.0$ \\
\hline INR ER & $1.28 \pm 0.65$ & $2.34 \pm 2.02$ \\
\hline Base excess ER & $-3.0 \pm 4.5$ & $-12.3 \pm 9.1$ \\
\hline Coagulopathy & $29.2 \%$ & $70.8 \%$ \\
\hline
\end{tabular}

The "Control group" comprises 36,391 patients who were treated in the ER (including 35,477 patients without any CPR and 944 patients after prehospital CPR)

$E R$ emergency room, $C P R$ cardiopulmonary resuscitation, ISS Injury Severity Score, AIS Abbreviated Injury Severity Score, GCS Glasgow Coma Score, BP blood pressure, TASH Trauma-Associated Severe Haemorrhage, INR international normalised ratio 
Table 2 Results of multivariate logistic regression analysis with resuscitation in the ER as dependent variable (Nagelkerke's $R^{2}=0.3$ ) are shown with $n=29,690$ including the $95 \% \mathrm{Cl}$

\begin{tabular}{|c|c|c|c|c|c|}
\hline & Koef & SE & $p$ value & OR & $95 \% \mathrm{Cl}$ \\
\hline Preclinical resuscitation & 1.747 & 0.107 & $<0.001$ & 5.74 & $4.65-7.07$ \\
\hline Preclinical catecholamines & 0.419 & 0.091 & $<0.001$ & 1.52 & $1.27-1.82$ \\
\hline Preclinical thorax drainage & 0.43 & 0.102 & $<0.001$ & 1.54 & $1.26-1.88$ \\
\hline Coagulopathy & 0.777 & 0.077 & $<0.001$ & 2.18 & $1.87-2.53$ \\
\hline Preclinical GCS $\leq 8$ & 0.248 & 0.081 & 0.002 & 1.28 & $1.87-2.53$ \\
\hline AIS thorax $\geq 3$ & 0.23 & 0.08 & 0.004 & 1.26 & $1.08-1.47$ \\
\hline AIS abdomen $\geq 3$ & 0.205 & 0.078 & 0.009 & 1.23 & $1.05-1.43$ \\
\hline AlS extremities $\geq 3$ & 0.236 & 0.073 & 0.001 & 1.27 & $1.10-1.46$ \\
\hline Preclinical shock (BP $\leq 90 \mathrm{mmHG}$ ) & 0.301 & 0.078 & $<0.001$ & 1.35 & $1.16-1.57$ \\
\hline Mild hypotension on admission (BP $\leq 90 \mathrm{mmHG}$ ) & 1.250 & 0.075 & $<0.001$ & 3.49 & $3.02-4.04$ \\
\hline Constant & -5.587 & 0.100 & $<0.001$ & 0.004 & \\
\hline
\end{tabular}

Statistical significant results are bold. All variables considered in the multivariate models are presented, and all were found to be significant

$E R$ emergency room, SE standard error, OR odds ratio, CI confidence interval, GCS Glasgow Coma Score, AIS Abbreviated Injury Severity Score, BP blood pressure,

statistic significant results are bold $(p \leq 0.005)$

moderate outcome (46 \% among the survivors). After resuscitation only in the ER, $25.6 \%$ survived in total and 19.2 $\%$ had a GOS of 4 or 5 ( $75.0 \%$ among the survivors). In total $4.8 \%$ in the group with preclinical and ER resuscitation survived and only $2.7 \%$ revealed a good or moderate outcome (56 \% among the survivors).

All three analysed subgroups of patients with resuscitation were statistically significant $(p<0.005$; $\mathrm{U}$ test $)$ in terms of good and moderate outcome.

Furthermore, we cross-tabulated the prognostic factors for patients with good and moderate outcome (GOS 4+5) after resuscitation in the ER, as illustrated in Table 4:

Results of multivariate logistic regression analysis with bad outcome (GOS 1-3) as a dependent variable in patients with preclinical resuscitation (Nagelkerke's $R^{2}=$ 0.28 ) are shown with $\mathrm{n}=841$ in Table 5 :

\section{Discussion}

Injury is the leading cause of death in industrialised countries of people aged between 1 and 44 years $[19,29,30]$, and trauma is the leading cause of death from 1 through 21 years of age [31]. Survival after traumatic out-of-hospital cardiopulmonary arrest in general seems to be rare in the last decades, even with maximum resuscitative efforts. Recently, a study introduced a trauma CPR algorithm and in their collective over $60 \%$ management errors and preventable deaths were found. They concluded that the prehospital trauma management has the highest potential to improve tCPR and survival [21].

In this study we analysed data from the TR-DGU. Our patient cohort consists of victims of severe blunt trauma expressed by a median ISS of 42.2, comparable to other studies in adults on TCRA [12, 32]. Little is known about specific and significant factors during the treatment of polytraumatised patients in terms of different time points of resuscitation (preclinical, in the emergency room or at both times) with specific focus on CPR in the ER. With ample data from the TR-DGU, we have been able to analyse factors and parameters in relation

Table 3 Results of the Glasgow Outcome Scale (GOS) [26] after resuscitation in the prehospital phase and/or in the ER based on 38,499 adult trauma patients

\begin{tabular}{|c|c|c|c|c|c|c|}
\hline Glasgow Outcome Scale & 1 Dead & 2 Vegetative state & 3 Severe disability & 4 Moderate disability & 5 Good recovery & $\begin{array}{l}\text { Good and moderate } \\
\text { outcome combined }\end{array}$ \\
\hline No resuscitation $(n=35,447)$ & $16.4 \%(5816)$ & $2.4 \%(865)$ & $12.7 \%(4495)$ & $25.4 \%(9015)$ & $43.0 \%(15,256)$ & $68.5 \%(24,271)$ \\
\hline $\begin{array}{l}\text { Prehospital resuscitation } \\
(\mathrm{n}=944)\end{array}$ & $68.3 \%(645)$ & $6.4 \%(60)$ & $10.6 \%(100)$ & $7.7(73)$ & $7.0 \%(66)$ & $14.7 \%(139)$ \\
\hline ER resuscitation $(n=1197)$ & $74.4 \%(891)$ & $1.3 \%(15)$ & $5.1 \%(61)$ & $9.7 \%(116)$ & $9.5 \%(114)$ & $19.2 \%(230)$ \\
\hline $\begin{array}{l}\text { Prehospital + ER resuscitation } \\
n=911)\end{array}$ & $95.2 \%(867)$ & $0.7 \%(6)$ & $1.4 \%(13)$ & $1.3 \%(12)$ & $1.4 \%(13)$ & $2.7 \%(25)$ \\
\hline
\end{tabular}

Pairwise comparison of the three different resuscitation subgroups ( $U$ test):

ER vs. prehospital: $p=0.007$

ER vs. prehospital + ER: $p<0.001$

Prehospital vs. prehospital + ER: $p<0.001$

$E R$ emergency room 
Table 4 Prognostic factors for patients with good and moderate outcome (GOS $4+5)$ after resuscitation in the ER using a cross-tabulation

\begin{tabular}{|c|c|c|c|c|}
\hline & $\begin{array}{l}\text { GOS } 4+5 \text { (good and moderate outcome) } \\
\text { in } \%\end{array}$ & $\begin{array}{l}\text { GOS 1-3 (severe disability, } \\
\text { vegetative state, dead) in \% }\end{array}$ & Total $n=$ & \\
\hline ER resuscitation & 90.2 & 52.2 & 1197 & $p<0.001$ \\
\hline Preclinical + ER resuscitation & 9.8 & 47.8 & 911 & \\
\hline Penetrating trauma & 8.9 & 7.8 & 163 & $p=0.53$ \\
\hline Blunt trauma & 91.1 & 92.2 & 1894 & \\
\hline Female patients & 27.2 & 30.1 & 628 & $p=0.38$ \\
\hline Male patients & 72.8 & 69.9 & 1483 & \\
\hline \multicolumn{5}{|l|}{ Type of injury } \\
\hline Car & 28.5 & 29.1 & 582 & $p=0.26$ \\
\hline Motorcycle & 14.6 & 13,6 & 275 & \\
\hline Bicycle & 6.9 & 7.1 & 142 & \\
\hline Pedestrian & 9.8 & 13.4 & 260 & \\
\hline Fall $>3 \mathrm{~m}$ & 17.9 & 20.1 & 397 & \\
\hline Fall $<3 \mathrm{~m}$ & 6.9 & 4.4 & 94 & \\
\hline Other & 15.4 & 12.2 & 253 & \\
\hline \multicolumn{5}{|l|}{ Severity of injury (AIS) } \\
\hline AlS head $<3$ & 56.0 & 39.5 & 881 & $p<0.001$ \\
\hline AlS head $\geq 3$ & 44.0 & 60.5 & 1241 & \\
\hline AIS thorax $<3$ & 31.5 & 25.7 & 560 & $p=0.05$ \\
\hline AIS thorax $\geq 3$ & 68.5 & 74.3 & 1562 & \\
\hline AlS abdomen $<3$ & 69.6 & 68.1 & 1449 & $p=0.67$ \\
\hline AIS abdomen $\geq 3$ & 30.4 & 31.9 & 673 & \\
\hline AIS extremities $<3$ & 50.6 & 53.8 & 1134 & $p=0.35$ \\
\hline AIS extremities $\geq 3$ & 49.4 & 46.2 & 988 & \\
\hline Preclinical RR >90 mmHG & 73.3 & 34.9 & 664 & $p<0.001$ \\
\hline Preclinical RR $\leq 90 \mathrm{mmHG}$ & 26.7 & 65.1 & 991 & \\
\hline $\mathrm{ER} R \mathrm{RR}>90 \mathrm{mmHG}$ & 69.2 & 31.4 & 647 & $p<0.001$ \\
\hline ER RR $\leq 90 \mathrm{mmHG}$ & 30.8 & 68.6 & 1130 & \\
\hline No blood transfusion & 52.5 & 33.9 & 700 & $p<0.001$ \\
\hline Blood transfusion & 47.5 & 66.1 & 1222 & \\
\hline$\leq 10$ blood transfusions & 79.8 & 72.8 & 1418 & $p=0.018$ \\
\hline$>10$ blood transfusions & 20.2 & 27.2 & 505 & \\
\hline No coagulopathy & 57.8 & 24.1 & 471 & $p<0.001$ \\
\hline Coagulopathy & 42.2 & 75.9 & & \\
\hline Preclinical GCS >8 & 70.2 & 23.8 & 593 & $p<0.001$ \\
\hline Preclinical GCS $\leq 8$ & 29.8 & 76.2 & 1420 & \\
\hline No acidosis & 57.7 & 20.2 & 313 & $p<0.001$ \\
\hline Acidosis & 42.3 & 79.8 & 925 & \\
\hline Age in years & $45 \pm 20$ & $50 \pm 21$ & 2122 & $p=0.005$ \\
\hline ISS & $30 \pm 12$ & $45 \pm 18$ & 2122 & $p<0.001$ \\
\hline Preclinical volume in $\mathrm{ml}$ & $1302 \pm 974$ & $1645 \pm 1173$ & 1886 & $p<0.001$ \\
\hline TASH score & $9.6 \pm 6.3$ & $14.6 \pm 5.6$ & 1047 & $p<0.001$ \\
\hline Hemoglobin in the TR & $11.2 \pm 2.9$ & $8.8 \pm 3.5$ & 1777 & $p<0.001$ \\
\hline
\end{tabular}


Table 4 Prognostic factors for patients with good and moderate outcome (GOS $4+5)$ after resuscitation in the ER using a cross-tabulation (Continued)

\begin{tabular}{|c|c|c|c|c|}
\hline Quick in the TR & $72 \pm 25$ & $47 \pm 51$ & 1449 & $p<0.001$ \\
\hline INR in the TR & $1.40 \pm 0.70$ & $2.51 \pm 2.14$ & 1482 & $p<0.001$ \\
\hline Base excess & $-6.2 \pm 7.1$ & $-13.2 \pm 9.0$ & 1238 & $p<0.001$ \\
\hline
\end{tabular}

GOS Glasgow Outcome Scale, ER emergency room, AIS Abbreviated Injury Severity Score, RR Riva Rocchi, GCS Glasgow Coma Score, ISS Injury Severity Score, TASH Trauma-Associated Severe Haemorrhage, $T R$ trauma room, INR international normalised ratio, statistic significant results are bold $(p \leq 0.005)$

to mortality and neurological outcome as well. Moreover, prognostic factors were analysed.

\section{Mortality}

The $25.6 \%$ survivor rate after resuscitation in the ER we calculated is much higher than the $3.3 \%$ reported in a systematic review containing a meta-analysis reporting a total of 238 survivors out of 5391 patients [13]; our study's survivor rate is also much higher than that in a retrospective analysis reporting $5.4 \%$ of 1114 patients surviving to hospital discharge after out-of-hospital traumatic cardiopulmonary arrest [33]. The results are similar to the $29 \%$ survivor rate in a prospective observation study from Berlin/Germany [21].

In another study, $5.0 \%$ of the patients underwent resuscitation during trauma room (TR) treatment [14]. In that study, the neurological outcome after only resuscitation in the ER was better than after preclinical resuscitation and after preclinical and ER resuscitation. One reason for this finding could be an optimal setting with an interdisciplinary team of specialists vs. a smaller preclinical team with normally only one physician. Another reason might be that cardiac arrest was witnessed in the ER setting in conjunction with resuscitation being initiated immediately, thus keeping the "downtime" minimal.

Moreover the highest rate of survivors was found in the group of preclinical resuscitation with $31.7 \%$. Unlike in the British and American paramedic systems, the

Table 5 Results of multivariate logistic regression analysis with bad outcome (GOS 1-3) as a dependent variable of the adults after preclinical resuscitation only (Nagelkerke's $R^{2}=0.28$ ) are shown with $n=841$

\begin{tabular}{llllll}
\hline & Koef & SE & $p$ value & OR & $95 \% \mathrm{Cl}$ \\
\hline Preclinical resuscitation & 1.131 & 0.258 & $\boldsymbol{p}<\mathbf{0 . 0 0 1}$ & 3.10 & $1.9-5.1$ \\
ISS (per point) & 0.048 & 0.007 & $\boldsymbol{p}<\mathbf{0 . 0 0 1}$ & 1.05 & $1.04-1.06$ \\
GCS $\leq 8$ preclinical & 1.357 & 0.186 & $\boldsymbol{p}<\mathbf{0 . 0 0 1}$ & 3.886 & $2.7-5.6$ \\
RR $\leq 90 \mathrm{mmHG}$ in the ER & 0.909 & 0.181 & $\boldsymbol{p}<\mathbf{0 . 0 0 1}$ & 2.481 & $1.74-3.54$ \\
Age $\geq 60$ years & 1.317 & 0.199 & $\boldsymbol{p}<\mathbf{0 . 0 0 1}$ & 3.732 & $2.53-5.52$ \\
Blood transfusion & 0.534 & 0.184 & $\boldsymbol{p}=\mathbf{0 . 0 0 4}$ & 1.706 & $1.189-2.446$ \\
Coagulopathy & 0.537 & 0.177 & $\boldsymbol{p}=\mathbf{0 . 0 0 2}$ & 1.711 & $1.210-2.420$ \\
Constant & 2.353 & 0.269 & $\boldsymbol{p}<\mathbf{0 . 0 0 1}$ & 0.95 & \\
\hline
\end{tabular}

GOS Glasgow Outcome Scale, SE standard error, OR odds ratio, CI confidence interval, ISS Injury Severity Score, GCS Glasgow Coma Score, RR Riva Rocchi, $E R$ emergency room, statistic significant results are bold $(p \leq 0.005)$ emergency physician in Germany (the Notarzt) stabilises the emergency patient at the scene of the accident. The emergency physician in Germany is qualified to declare patients dead at the scene, which is not included in this study. In some countries, paramedic systems cannot declare someone dead without consulting a physician. Thus some trauma victims are transported to the trauma centre just to be declared dead by the trauma physician on call. These differences make comparison with the prehospital phase of other countries' systems and with international studies therefore difficult.

On-scene triage is done by physicians and is based on their clinical assessment of the patient. These physicians also initiate the early treatment of haemorrhagic shock, provide respiratory assistance, manage pain and sedation, and insert chest tubes if necessary and can also use catecholamines (for sufficient cerebral perfusion, neurogenic shock, resuscitation, etc.). The results from the initial assessment of the patient's condition and those together with the patient's response to treatment are used by the dispatching physician to find the closest and best-suited facility for the patient. This organisation ensures the availability of resources at the receiving centre and avoids unnecessary secondary transfers from an overcrowded or poorly suited institution (accessed initially because of its proximity to the trauma scene). The cohort demographics including injury patterns are typical for western countries.

Our data fail to support data from earlier investigations by Fulton et al., who emphasised that the location of arrest (on scene, transport or emergency room) and age did not correlate with survival [34]. Our cohort's survival rates were different after preclinical resuscitation (31.7\%), resuscitation only in the ER (25.6\%), and after preclinical and ER resuscitation (4.8\%). A limitation of the study is that the patients who were dead at the scene were not included in the investigation, because these data are not available.

Our logistic regression model revealed that preclinical resuscitation followed by ISS, preclinical catecholamines, preclinical thorax drainage, coagulopathy, preclinical GCS $\leq 8$ points, AIS thorax, abdomen and extremities $\geq 3$ points, mild hypotension with $\leq 90 \mathrm{mmHg}$ during the preclinical or ER treatment are strongly predictive for mortality after resuscitation in the ER, in line with findings by Pickens et al., who reported that respiratory 
efforts and a GCS $\geq 4$ points at the scene have a positive influence on survival, and Durham et al., who reported the same regarding prehospital intubation [20,35]. Fulton et al. also noted that a loss of neurological function is associated with mortality [34].

The percentage of thoracic trauma in polytrauma patients is generally quite high, ranging from 34 to $70 \%[29,36-$ 39]. Huber-Wagner et al. observed that prehospital chest tube insertion was a strong predictor for survival. Also tension pneumothorax is known as the most common reason for definitive preventable traumatic death [17].

They therefore advised on-scene chest decompression of TCRA patients in case of the decision to initiate emergency cardiac care (ECC) [40].

In the European Resuscitation Council Guidelines for Resuscitation 2015, bilateral chest decompression is advised in the algorithm to address one of the reversible causes for resuscitation [41].

A study by Pickens et al. reported that several survivors met criteria for non-treatment according to proposed clinical guidelines, and that insufficient prehospital treatment was the result [20]. Katz et al., for example, analysed a 25 $\%$ rate of misplaced tracheal tubes by paramedics (27/108) [42]. Furthermore, Cera et al. found that the time of intubation (on-scene versus in-hospital) did not influence survival [1].

Normally, patients in a clinical setting should never be deprived of sufficient oxygen for a relevant period of time, unlike what can happen in a preclinical setting, where patients probably suffer several minutes without oxygenation before the medical staff reaches the scene. The fact that a patient requires resuscitation in a preclinical and ER setting seems to reflect the trauma's severity and according to the literature often appears to be associated with a poor outcome.

In our investigation among $31.7 \%$ survivors in the group of preclinical resuscitation $46 \%$ had a good or moderate neurological outcome; after resuscitation in the ER $25.6 \%$ survived, with $19.2 \%$ having a GOS 4 or 5 .

GOS has been documented in the TR-DGU since 2002, thus there are no GOS records for patients from 1993 to 2001. Our GOS calculation is therefore based on 38499 patients.

Reliable data describing outcomes in detail and including neurological function are rare in the literature. A recently published literature review covering 1980 to 2011 analysed the outcomes of children treated for out-ofhospital traumatic cardiopulmonary arrest who reached the clinic alive: 29 patients (57 \%) were severely disabled; 3 children $(6 \%)$ moderately disabled, and 19 patients (37\%) had a normal outcome [43]. Margolin et al provide information on the GOS in six of 13 survivors (46.2 $\%)$ out of 67 patients (79.1 \% blunt trauma) who had at least a satisfactory outcome as expressed by a GOS of $\geq 4$ [32]. Other studies describe patients' outcome data in terms of their being "neurologically intact" after TCRA: Fialka et al. observed $100 \%$ of patients surviving with no neurological deficit $(4 / 4, \mathrm{n}=38)$ [44], Fulton et al. $83.3 \%$ of survivors being neurologically intact $(5 / 6, \mathrm{n}=$ 245) [34], Powell et al. $81.0 \%$ of survivors $(21 / 26, n=$ 959) [11], Stratton et al. $66.6 \%(3 / 9, \mathrm{n}=79)$ [8], Battistella et al. $56.3 \%(9 / 16, \mathrm{n}=604)$ [6], Branney et al. $3.9 \%$ $(34 / 42, n=950)$ [45], and Martin et al. no neurologically intact patients $(0 / 1, \mathrm{n}=110)$ [12].

Yanagawa et al. detected no significant differences between out-of-hospital TCRA patients with or without head injury except for the fact that the blunt traumatic patients with a head injury more often displayed a return to spontaneous circulation (ROSC). However, they observed no head-injury effect on the outcome [3]. In our investigation, a preclinical GCS $\leq 8$ had a significant odds ratio of 2.18 in the multivariate logistic regression analysis with resuscitation in the ER as a dependent variable. Moreover, an AIS head $\geq 3$ was a significant prognostic factor in our cross-tabulation, with a GOS 4 and 5 and resuscitation in the ER and preclinical GCS $\leq 8$ showing a significant odds ratio of 3.886 in multivariate logistic regression analysis with GOS 1-3 as a dependent variable.

Our multivariate logistic regression revealed that injury severity, the preclinical use of catecholamines and preclinical thorax drainage, coagulopathy, a preclinical GCS $\leq 8$, severe injuries to the thorax, abdomen and extremities, and preclinical shock in the ER are strongly predictive of resuscitation in the ER.

Sorted according to a bad prognosis with GOS 1-3, these factors were preclinical resuscitation, ISS, preclinical GCS $\leq 8$, mild hypotension in the ER with Riva Rocchi (RR) $\leq 90 \mathrm{mmHG}$, blood transfusion and catecholamines, and age $\geq 60$ years.

Base deficit is a rapidly and widely available serum laboratory marker of systemic acidosis that increases with hypoxemia and/or shock. In the trauma setting, the base deficit correlates with the blood-transfusion requirement, risks of multiorgan failure and mortality in adult patients, including those with traumatic brain injury [31-33].

These findings are in line with the sparse data in the literature reporting that blood pressure $=0$ [34] or low blood pressure in the field were prognostic factors [35].

\section{Limitations}

Our study has several limitations. Patients declared dead on-scene without transportation to a hospital were not recorded in the database. Moreover, only patients surviving CPR and transportation to the hospital could be included. That means that our database does not include those patients who required prehospital CPR but did not 
achieve ROSC. This is because the TraumaRegister $\mathrm{DGU}^{\bullet}$ was established as a tool for quality assessment in the acute care hospital. Patients who died before admission were thus not relevant to such an assessment. Furthermore, Germany maintains no documentation system that covers all trauma cases, including all prehospital deaths. Our survival rates thus do not refer to all cases with cardiac arrest after trauma but only to those cases who were admitted to the acute care hospital alive, i.e., with ROSC. This limitation and bias must be kept in mind when interpreting the surprisingly good survival rates.

This and other exclusion criteria (ISS $<16$ and secondarily transferred patients) were excluded. This could have biased the results.

Due to the fact that ECG data are not documented in the TR-DGU ${ }^{\circ}$, no information on cardiac rhythm was available. Furthermore, we had no specific information on the exact duration of chest compression in the pre- and/or in-hospital phase. According to the literature, resuscitation lasting more than 20 minutes seems to be associated with poor neurological outcome [33]. We could only analyse patients who underwent closed-chest compression on-scene or during transport and/or during treatment in the ED. The main limitation is our patient cohort's preselection. The TR-DGU ${ }^{\bullet}$ only includes patients who have been transported to a hospital after chest compression after TCRA. This might be one reason for the relatively high survival rates compared to other studies. The group investigated in our study represents patients who had been resuscitated because of a subjective assessment by the on-scene emergency physician who perceived a relatively certain chance of survival for those patients.

\section{Conclusions}

According to our data, CPR in patients after severe trauma seems to yield a better outcome than most studies have reported, and it appears to be more justified than the latest guidelines would imply. The results potentially alter the attitude towards the usefulness of intensive resuscitation in patients suffering trauma.

Prehospital resuscitation is associated with higher survival rate compared with resuscitation in the ER. Overall, good and moderate neurological outcomes were highest in the group of only ER resuscitation and among the survivors in the prehospital resuscitation group.

Resuscitation in both the preclinical phase and the ER had fatal mortality rate, even though among the survivors the rate of patients with a GOS 4 and 5 was quite high. We hope that our findings will help future patients by facilitating the drafting of guidelines and algorithms for the resuscitation of polytraumatised patients.

\section{Key messages}

- Patients' outcomes after severe trauma seems to be better than those reported in most previous studies.

- Resuscitation in the ER is associated with better neurological outcomes compared with resuscitation in a preclinical context or in both the preclinical phase and the ER.

- Among the survivors, patients who survived preclinical resuscitation had the highest rate of good or moderate outcome

- These data might support algorithms and guidelines in the future dealing with the resuscitation of polytraumatised patients.

\section{Acknowledgements}

The article processing charge was funded by the German Research Foundation (DFG) and the Albert Ludwigs University Freiburg in the funding programme Open Access Publishing.

\section{Funding}

No funding to declare.

\section{Availability of data and materials}

The datasets during and/or analysed during the current study are available from the corresponding author on reasonable request.

\section{Authors' contributions}

JZ made substantial contributions to the design of the study and the acquisition of data and analysis of data. RL made substantial contributions to analysis and interpretation of data and statistical tests performed. All authors have made substantial contributions to all of the following: the conception of the study, interpretation of the data, drafting the manuscript or revising it critically for important intellectual content. All authors read and approved the final manuscript.

\section{Competing interests}

The authors declare that they have no competing interests.

\section{Consent for publication}

Not applicable.

\section{Ethics approval and consent to participate}

The TraumaRegister DGU ${ }^{\circledR}$ of the German Trauma Society was founded in 1993. The aim of this multi-centre database is the anonymous and standardised documentation of severely injured patients. Data are collected prospectively in four consecutive time periods from the site of the accident until discharge from hospital: (A) prehospital, (B) emergency room, (C) intensive care unit, and (D) discharge. The inclusion criterion is admission to hospital via the emergency room with subsequent ICU care or reaching the hospital with vital signs and dying before admission to the ICU. The participating hospitals submit their data anonymously to a central database via a web-based application.

Data anonymity is guaranteed both for the individual patient and participating hospital. The TraumaRegister DGU ${ }^{\circledast}$ comprises epidemiological, physiological, laboratory, diagnostic, operative, interventional and intensive care data as well as scoring and outcome data. The Ethics Commission at the University of Freiburg Medical Centre confirmed that no ethical approval for this retrospective analysis of anonymous routine data is necessary (Reference Nr.:10003/15).

\section{Author details}

'Department of Orthopedics and Trauma Surgery, Freiburg University Hospital, Hugstetter Str. 55, 79098 Freiburg, Germany. ${ }^{2}$ Institute for Research in Operative Medicine (IFOM), University of Witten/Herdecke, Herdecke, Germany. ${ }^{3}$ Clinic of Orthopedics and Trauma Surgery, Sozialstiftung Bamberg, Bamberg, Germany. 
Received: 14 June 2016 Accepted: 22 August 2016

\section{Published online: 06 September 2016}

\section{References}

1. Cera SM, et al. Physiologic predictors of survival in post-traumatic arrest. Am Surg. 2003;69(2):140-4

2. Rosemurgy AS, et al. Prehospital traumatic cardiac arrest: the cost of futility. J Trauma. 1993;35(3):468-73. discussion 473-4.

3. Yanagawa $Y$, et al. Experience of treatment for blunt traumatic out-ofhospital cardiopulmonary arrest patients over 24 years: head injury v.S. non-head injury. No Shinkei Geka. 2004;32(3):231-5.

4. Shimazu S, Shatney $\mathrm{CH}$. Outcomes of trauma patients with no vital signs on hospital admission. J Trauma. 1983;23(3):213-6.

5. Bouillon B, et al. Trauma and circulatory arrest. 224 preclinical resuscitations in Cologne in 1987-1990. Anaesthesist. 1994:43(12):786-90.

6. Battistella FD, et al. Field triage of the pulseless trauma patient. Arch Surg. 1999;134(7):742-5. discussion 745-6.

7. Pasquale MD, et al. Defining "dead on arrival": impact on a level I trauma center. J Trauma. 1996:41(4):726-30.

8. Stratton SJ, Brickett K, Crammer T. Prehospital pulseless, unconscious penetrating trauma victims: field assessments associated with survival. J Trauma. 1998:45(1):96-100.

9. Soar J, et al. European Resuscitation Council guidelines for resuscitation 2005. Section 7. Cardiac arrest in special circumstances. Resuscitation. 2005;67 Suppl 1:S135-70.

10. Stockinger ZT, McSwain Jr NE. Additional evidence in support of withholding or terminating cardiopulmonary resuscitation for trauma patients in the field. J Am Coll Surg. 2004;198(2):227-31.

11. Powell DW, et al. Is emergency department resuscitative thoracotomy futile care for the critically injured patient requiring prehospital cardiopulmonary resuscitation? J Am Coll Surg. 2004;199(2):211-5.

12. Martin SK, et al. Blunt trauma patients with prehospital pulseless electrical activity (PEA): poor ending assured. J Trauma. 2002;53(5): 876-80. discussion 880-1.

13. Zwingmann J, et al. Survival and neurologic outcome after traumatic out-ofhospital cardiopulmonary arrest in a pediatric and adult population: a systematic review. Crit Care. 2012:16(4):R117.

14. Zwingmann J, et al. Outcome and risk factors in children after traumatic cardiac arrest and successful resuscitation. Resuscitation. 2015;96:59-65.

15. Sauaia A, et al. Epidemiology of trauma deaths: a reassessment. J Trauma. 1995:38(2):185-93.

16. Kleber C, et al. Overall distribution of trauma-related deaths in Berlin 2010: advancement or stagnation of German trauma management? World J Surg. 2012;36(9):2125-30.

17. Kleber C, et al. Trauma-related preventable deaths in Berlin 2010: need to change prehospital management strategies and trauma management education. World J Surg. 2013;37(5):1154-61.

18. Velmahos GC, et al. Outcome of a strict policy on emergency department thoracotomies. Arch Surg. 1995;130(7):774-7.

19. Hopson $L R$, et al. Guidelines for withholding or termination of resuscitation in prehospital traumatic cardiopulmonary arrest: joint position statement of the National Association of EMS Physicians and the American College of Surgeons Committee on Trauma. J Am Coll Surg. 2003;196(1):106-12.

20. Pickens JJ, Copass MK, Bulger EM. Trauma patients receiving CPR: predictors of survival. J Trauma. 2005;58(5):951-8.

21. Kleber $C$, et al. Requirement for a structured algorithm in cardiac arrest following major trauma: epidemiology, management errors, and preventability of traumatic deaths in Berlin. Resuscitation. 2014;85(3):405-10.

22. Mollberg NM, et al. The consequences of noncompliance with guidelines for withholding or terminating resuscitation in traumatic cardiac arrest patients. J Trauma. 2011;71(4):997-1002.

23. Ruchholtz $\mathrm{S}$, et al. The trauma register of the 'Polytrauma' Committee of the German Society of Trauma Surgery as the basis for quality management in the management of severely injured patients. Langenbecks Arch Chir Suppl Kongressbd. 1997;114:1265-7.

24. Ruchholtz S. The Trauma Registry of the German Society of Trauma Surgery as a basis for interclinical quality management. A multicenter study of the German Society of Trauma Surgery. Unfallchirurg. 2000;103(1):30-7.

25. Ruchholtz S, S. German Society of Trauma. External quality management in the clinical treatment of severely injured patients. Unfallchirurg. 2004; 107(10):835-43.
26. Jennett B, Bond M. Assessment of outcome after severe brain damage. Lancet. 1975:1(7905):480-4.

27. Yucel N, et al. Trauma Associated Severe Hemorrhage (TASH)-Score: probability of mass transfusion as surrogate for life threatening hemorrhage after multiple trauma. J Trauma. 2006;60(6):1228-36. discussion 1236-7.

28. Maegele $M$, et al. Early coagulopathy in multiple injury: an analysis from the German Trauma Registry on 8724 patients. Injury. 2007;38(3):298-304.

29. Zwingmann J, et al. Injury patterns in polytraumatized children and consequences for the emergency room management. Acta Chir Orthop Traumatol Cech. 2010;77(5):365-70.

30. Zwingmann J, et al. Injury severity and localisations seen in polytraumatised children compared to adults and the relevance for emergency room management. Zentralbl Chir. 2008;133(1):68-75.

31. American Academy of Pediatrics. Committee on Child Abuse and Neglect and Committee on Bioethics. Foregoing life-sustaining medical treatment in abused children. Pediatrics. 2000;106(5):1151-3.

32. Margolin DA, et al. Response after out-of-hospital cardiac arrest in the trauma patient should determine aeromedical transport to a trauma center. J Trauma. 1996;41(4):721-5.

33. American College of Surgeons Committee on Trauma, et al. Withholding or termination of resuscitation in pediatric out-of-hospital traumatic cardiopulmonary arrest. Pediatrics. 2014;133(4):e1104-16.

34. Fulton RL, Voigt WJ, Hilakos AS. Confusion surrounding the treatment of traumatic cardiac arrest. J Am Coll Surg. 1995;181(3):209-14.

35. Durham 3rd LA, et al. Emergency center thoracotomy: impact of prehospital resuscitation. J Trauma. 1992:32(6):775-9.

36. Bardenheuer $M$, et al. Epidemiology of the severely injured patient. A prospective assessment of preclinical and clinical management. AG Polytrauma of DGU. Unfallchirurg. 2000;103(5):355-63.

37. Gatzka C, et al. Injury pattern and clinical course of children with multiple injuries in comparison to adults, Ab 11-year analysis at a clinic of maximum utilization. Unfallchirurg. 2005;108(6):470-80

38. Reichmann I, et al. Comparison of severe multiple injuries in childhood and adulthood. Unfallchirurg. 1998;101(12):919-27.

39. Schalamon J, et al. Multiple trauma in pediatric patients. Pediatr Surg Int. 2003;19(6):417-23.

40. Huber-Wagner $\mathrm{S}$, et al. Outcome in 757 severely injured patients with traumatic cardiorespiratory arrest. Resuscitation. 2007;75(2):276-85.

41. Truhlar A, et al. European Resuscitation Council Guidelines for Resuscitation 2015: Section 4. Cardiac arrest in special circumstances. Resuscitation. 2015; 95:148-201.

42. Katz SH, Falk JL. Misplaced endotracheal tubes by paramedics in an urban emergency medical services system. Ann Emerg Med. 2001;37(1):32-7.

43. American College of Surgeons Committee on Trauma, et al. Withholding or termination of resuscitation in pediatric out-of-hospital traumatic cardiopulmonary arrest. Ann Emerg Med. 2014;63(4):504-15.

44. Fialka C, et al. Open-chest cardiopulmonary resuscitation after cardiac arrest in cases of blunt chest or abdominal trauma: a consecutive series of 38 cases. J Trauma. 2004;57(4):809-14.

45. Branney SW, et al. Critical analysis of two decades of experience with postinjury emergency department thoracotomy in a regional trauma center. J Trauma. 1998;45(1):87-94. discussion 94-5.

\section{Submit your next manuscript to BioMed Central and we will help you at every step:}

- We accept pre-submission inquiries

- Our selector tool helps you to find the most relevant journal

- We provide round the clock customer support

- Convenient online submission

- Thorough peer review

- Inclusion in PubMed and all major indexing services

- Maximum visibility for your research

Submit your manuscript at www.biomedcentral.com/submit 